\title{
Adaptação e validação da Escala de Senso de Humor Situacional
}

\author{
Adriana Beneides Scares - UniversidadeSalgadbdeOlivera, Niterá, RiodeJaneiro, Brasil \\ Luaiana Marão- UnivesidadeSalgadodeOliveira, Niteái, RiodeJanero, Brasil
}

\begin{abstract}
Resumo
O estudo visou desenvolver uma versão em português brasileiro do Situational Humr ResponßeQuestionaire(SHRQ), com adaptação transcultural e avaliação das medidas psicométricas em amostra brasileira. 0 instrumento em português de Portugal foi vertido para o português brasileiro passando pelas etapas de adaptação semântica, comparação das versões, validade de face e de conteúdo. 0 instrumento foi aplicado a 621 sujeitos. Foi feita extração de fatores com rotação oblíqua, análise das cargas fatoriais e consistência interna. Foram apontados três fatores: humor com amigos e colegas $(\alpha=0,70)$; influências sobre o humor $(\alpha=0,70)$; e humor em situações adversas $(\alpha=0,55)$. A consistência interna sugere que para 0 contexto brasileiro podem ser aperfeiçoados os itens de forma a aumentar o grau de precisão do fator. Contudo, 0 instrumento apresenta consistência interna aceitável.
\end{abstract}

Palavasdhave Humor; Avaliação psicológica; Psicometria.

\section{Adaptation and validation of the Situational Humor Response Questionaire}

\begin{abstract}
This study aimed to develop a Brazilian Portuguese version of the Situational Humor Response Questionnaire (SHRQ), with transcultural adaptation and evaluation of the psychometric measures for the Brazilian context. The instrument has been translated from the original Portuguese to the Brazilian Portuguese, respecting the stages of semantic adaptation, the comparison of the versions and the adaptation to the cross-cultural in face validity and content. The instrument was applied to a sample of 621 participants. It has extracted factors with oblique rotation, analysis of factorial loadings and internal analysis of consistency. The results have pointed out three main factors: mood with friends and colleagues $(\alpha=0.70)$; influences on moods $(\alpha=0.70)$, and humor in adverse situations $(\alpha=0.55)$. The internal consistency suggests that for the Brazilian context some items can be improved, especially concerning the humor in adverse situations, in order to enhance the precision factor. However, it provides an acceptable internal consistency.
\end{abstract}

Keguads Humor; Psychological assessment; Psychometrics.

\section{Adaptación y validez de la Escala de Sentido del Humor Situacional}

\section{Resumen}

El estudio visó desarrollar una versión en portugués brasileño del Situational Humr Response Questianaire(SHRQ), con adaptación transcultural y evaluación de las medidas psicométricas en muestra brasileña. El instrumento en portugués de Portugal fue traducido para el portugués brasileño pasando por las etapas de adaptación semántica, comparación de las versiones, validez facial y de contenido. El instrumento fue aplicado en 621 sujetos. Fue hecha la extracción de factores con rotación oblicua, análisis de las cargas factoriales y consistencia interna. Fueron apuntados tres factores: humor con amigos y compañeros $(\alpha=0,70)$; influencias sobre el humor $(\alpha=0,70)$; y humor en situaciones adversas $(\alpha=0,55)$. La consistencia interna sugiere que para el contexto brasileño pueden ser perfeccionados los ítems para aumentar el grado de precisión del factor. Sin embargo, el instrumento presenta consistencia interna aceptable.

Palabrasdave Humor; Evaluación psicológica; Psicometria.

Matérias de jomais e entrevistas com diversos tipos de profissionais - como médicos, psicólogos, psicanalistas, sociólogos têm apontado o senso de humor como uma forma positiva de lidar com 0 estresse nos tempos atuais. Segundo Dixon (1980), o humor é uma importante estratégia humana para lidar eficazmente com o estresse, porém poucos são os instrumentos que permitem mensurar o senso de humor das pessoas. Além dos aspectos individuais relacionados ao bem-estrar e à saúde, estudos mostram também as funções sociais do humor nas relações interpessoais (D uncan, 1984; Morrell, 1997) e, segundo Brief e Weiss (2002), há mais de meio século os pesquisadores organizacionais começaram a demonstrar um interesse maior sobre humor e emoções no ambiente de trabalho.

Segundo Houzel (2002), centros da linguagem no lobo temporal podem ser ativados por anedotas relacionadas com 0 significado das palavras, 0 que teria sido indicado em pesquisas de mapeamento de zonas ativas do cérebro. Segundo o autor, os trocadilhos ativam o córtex pré-frontal medial ventral, processando sinapses relacionadas com o som das palavras.

Silva-Filho (2003) critica a pouca consideração da parte psicológica (emocional) como explicação para 0 ato de "achar graça". Para 0 autor, o riso ocorreria quando um impulso reprimido sexual ou agressivo foge subitamente da censura e seria uma forma de as pessoas sentirem-se superiores e no riso descarregarem a agressividade. No instrumento de Humor Situacional, por exemplo, várias situações são apresentadas como vividas pelo sujeito como sem saída, ou seja, não há nada que se possa fazer naquela situação para evitar um desfecho desagradável a não ser interpretá-lo com humor, seja ele positivo, seja negativo. 
De acordo com D el Nero (1997), as emoçöes, os afetos, os sentimentos e os humores seriam conceitos equivalentes, do campo da emoção, que se distinguem dos conceitos de cognição (pensamento) e volição (vontade). 0 autor considera que dentre as funções da mente estão a consciência, a vontade, o pensamento, a emoção, a memória, 0 aprendizado, a imagem, a criatividade e a intuição.

Já Gondim e Siqueira (2004) baseiam-se na diferenciação apontada por Gray e Watson (2001) para emoção, humor, temperamento e afeto. Segundo os autores 0 afeto seria a integração de emoções e humores, que variam do temperamento no que tange à duração, ao objeto e ao estado. Assim, o humor teria uma duração maior que a da emoção (que duraria alguns segundos) e menor que a do temperamento (que duraria meses ou anos). Além disso, o humor teria um objeto difuso, enquanto a emoção teria um objeto focado. E em termos de estado, a emoção seria breve, 0 humor longo e o temperamento duradouro. Esses autores consideram que os estados afetivo-emocionais constituiriam uma categoria mais ampla, que incluiria duas subcategorias: emoções e afetos, estes últimos abarcando "os humores e os temperamentos, que teriam em comum sua persistência no tempo e sua relação com aspectos cognitivos" (p. 211).

Segundo Brief e Weiss (2002), os estados afetivos podem influenciar uma variedade de resultados de desempenho relevantes, incluindo julgamentos, respostas comportamentais, criatividade, comportamento de ajuda e tomada de risco. Os autores alertam para 0 fato de pesquisas em psicologia social mostrarem que estar em um estado de humor positivo em geral incentiva o comportamento de ajuda e cooperação e diminui a agressão. O humor positivo seria aquele que permite gerar alegria, melhorar as relações interpessoais e flexibilizar momentos de estresse e tensão. 0 humor negativo, por sua vez, seria aquele que leva a crítica, ao preconceito, podendo inclusive deteriorar relações sociais. No instrumento de humor situacional alguns itens procuram investigar inclusive o quanto os sujeitos valorizam estados de humor, buscando fazer amigos e conviver com pessoas que sejam bem-humoradas e que possam tornar a vida mais agradável.

Segundo Isen (1999), estar em um estado de espírito positivo pode melhorar a resolução criativa de problemas, o que foi confirmado em alguns estudos empíricos (Estrada, Isen \& Young, 1997; Madjar, Oldham \& Pratt, 2002). O humor também pode afetar negociações. Segundo pesquisa de Pillutla e Murnighan (1996), reações de raiva podem levar os negociadores a rejeitar propostas de grande interesse comercial, evidenciando que os julgamentos não são puramente baseados em aspectos cognitivos, nos quais as decisões seriam tomadas considerando-se 0 que é mais vantajoso em termos de padrões econômicos.

$\mathrm{Na}$ área de trabalho, estudo apontou para o poder de predição do humor positivo sobre o absenteísmo e que o humor positivo e negativo seriam preditores das intenções de abandono do emprego (George, 1989). Em outro estudo, tanto o estado de humor negativo como, sobretudo, o positivo seriam preditores do absenteísmo, mas apenas os estados de humor negativos seriam preditores do volume de negócios subsequentes (Pelled \& Xin, 1999).

O humor chama a atenção das pessoas pelo seu aspecto inesperado e promove um estado de espírito mais relaxado, fazendo com que as pessoas se comuniquem de forma mais descontraída e eficiente, reduzindo defesas individuais naturais em diferentes situações sociais, especialmente aquelas que envolvem elementos desagradáveis, tais como a aceitação e administração de erros, pedidos de desculpas, comunicação de notícias ruins, etc. (Morrell, 1997). Apesar da importância dos estudos sobre humor que, como aponta Morrell (1997), contribuem para a promoção da saúde, para a diminuição do estresse, para 0 aumento da flexibilidade cognitiva, para a facilitação da realização de negócios, raros são os instrumentos de medida encontrados para a aferição desses diferentes aspectos. $\mathrm{O}$ humor, segundo esse autor, é também um facilitador das relações interpessoais e, nesse sentido, 0 Situational Humr ResponseQuestionaire(SHRQ ) é o único instrumento validado encontrado hoje na literatura em português para medir quantitativamente 0 humor situacional que contextualiza as relações interpessoais.

$\mathrm{O}$ instrumento foi originalmente desenvolvido e validado em língua francesa, no Canadá, por Martin e Lefcourt (1984), e traduzido e validado para o português de Portugal por Caetano (1989), não tendo ainda aplicação em amostra brasileira. Assim, 0 objetivo deste estudo consiste na adaptação e na validação do instrumento para o português do Brasil.

\section{Método}

A pesquisa teve um total de 621 participantes, residentes no estado do Rio de Janeiro, tendo que ser excluídos dois casos em função da existência de dados faltantes, que impossibilita a utilização do instrumento de medida de forma adequada. A média de idade dos participantes foi de 34 anos ( $\mathrm{DP}=16,7 ; 17$ a 90 anos), $62,5 \%$ dos quais eram do sexo feminino. Os participantes foram caracterizados quanto a classe social, sendo $24 \%$ pertencentes à classe A, quase $50 \%$ à classe $\mathrm{B}$ e $25 \%$ à classe $\mathrm{C}$. Em relação ao estado civil, $58 \%$ eram solteiros e $30,2 \%$ casados. A amostra se 
dividiu em $49 \%$ profissionais e $51 \%$ estudantes universitários, dos quais $71 \%$ não tinham filhos.

0 instrumento utilizado para a pesquisa foi a versão adaptada para o português brasileiro do instrumento Situational Humr Response Questionaire (SHRQ), adaptado para o português por Caetano (1989). 0 instrumento continha inicialmente 21 itens, 18 deles relativos à mensuração da reação das pessoas a situações diversas, um item focado para a medição do quanto o humor é um aspecto valorizado na escolha dos amigos, outro item objetivando medir como a pessoa se classifica quanto à capacidade de se divertir e rir e uma última para mensurar o quanto as diversas situações podem influenciar na variação de humor. Os 18 itens que medem 0 senso de humor são apresentados de maneira contextualizada (e.g.: Se você estivesse fazendo compras numa cidade distante e, inesperadamente, visse um colega de escola (ou de trabalho) você...) numa escala de cinco pontos que varia de não achar nenhuma graça a rir abertamente.

0 processo de tradução e validação seguiu recomendações da literatura especializada (Cunha, 2001; Guillemin, 1995; Sperber, 2004). A linguagem foi inicialmente adaptada pelas pesquisadoras, que conheciam os objetivos e conceitos subjacentes ao estudo e que possuíam como língua-mãe o português brasileiro, criando-se uma primeira versão do instrumento. Após, essa versão foi enviada a três lusobrasileiros de diferentes áreas, que compararam as versões do instrumento com o português de Portugal e 0 português do Brasil, verificando equivalências semântica, idiomática, cultural e conceitual, gerando uma versão consensual denominada segunda versão. Em seguida, o instrumento foi aplicado a um grupo de 15 brasileiros não participantes das etapas anteriores, para validação semântica e ajustes na redação dos itens e das instruções. Os participantes foram incentivados a apontar suas dúvidas e dificuldades para responder ao instrumento, e suas avaliações foram centradas na validade de conteúdo e na validade de face, gerando, assim, a terceira versão da escala.

A validação psicométrica foi realizada a partir dessa terceira versão da escala. A amostra totalizou 25 respondentes por item e mais de 200 respondentes por fator medido, atendendo de forma plena às recomendações de Crocker e Algina (1986) e de Pasquali (1999) para tamanhos de amostra para análise fatorial.

Os dados foram coletados entre março e maio de 2010, com questionários autoaplicáveis, entregues e recolhidos dos respondentes, que foram informados sobre os objetivos da pesquisa e assinaram termo de consentimento. A aplicação dos questionários foi feita em diferentes ambientes, tais como locais de trabalho, escritórios, empresas, clínicas e universidades. Foram realizadas análises psicométricas, com 0 objetivo de avaliar sua validade e confiabilidade.

Os testes estatísticos foram efetuados com auxílio do programa SPSS versão 17.0. Para estimativa da confiabilidade (reiability) foram calculados 0 alfa de Cronbach e o lambda 2 de Gutman, sendo desejado valores iguais ou superiores a 0,70 em ambos os indicadores. Para a extração dos fatores, utilizaram-se a conjugação das técnicas de G1 (éngenvaluesuperior a 1), o teste visual do screeplat, a análise paralela e a análise de consistência teórica da solução fatorial. Para a análise da pertinência dos itens, adotou-se o critério de aceitação, comumente utilizado, de cargas fatoriais iguais ou superiores a 0,32 (Pasquali, 2003; Tabachnick \& Fidell, 2007). Os resultados obtidos são apresentados na seção que seguinte.

\section{Resultados e discussão}

O teste de validação semântica identificou que os brasileiros tinham dificuldade ao responder 0 item 21, o qual questionava: "Em que medida você varia de uma situação para outra na frequência que ri ou responde com humor ( $\mathrm{E}$ em que medida isso depende de com quem você está, de onde está, de como se sente, etc.)?”. Mais de um respondente afirmou que a resposta não seria a mesma se ele considerasse a companhia, o lugar ou como se sentia, ou seja, o contexto. Então, decidiuse por separar essa questão em três itens, os quais especificavam a variação de acordo com a companhia, o lugar ou como a pessoa se sentia. 0 restante do instrumento foi preservado, tendo sido feitas as adaptações de linguagem e contexto necessárias.

As respostas válidas foram, então, primeiramente submetidas a análises descritivas. 0 pressuposto da normalidade, embora não seja uma exigência - por ser a análise fatorial uma técnica de análise robusta -, também foi testado, pois os dados tendem a apresentar melhor configuração quando tal pressuposto é atendido. O critério utilizado para avaliar a normalidade da distribuição de dados foi ancorado na simetria, considerando-se uma distribuição normal sempre que o escore Z para simetria (simetria/ erro padrão de medida da simetria) foi maior que 1,96 (Tabachnick \& Fidell, 2007).

Em seguida, foram realizadas análises preliminares para verificar a fatorabilidade da matriz de correlações e análises fatoriais exploratórias (Prinapal Axis Factoing para estimar o número de fatores e definir a estrutura fatorial do instrumento. As análises relativas à quantidade de respostas em branco aos 24 itens analisados não ultrapassou a margem de $1 \%$, e não apresentou nenhum padrão de ocorrência, portanto, 
adotou-se o tratamento paurwsepara dados omissos.

A fatorabilidade da matriz de dados foi confirmada pelo indicador Kaiser-Meyer-Olkin Measure of Sampling Adequag $(\mathrm{KMO}=0,75)$ e pelo Teste de Esfericidade de Bartlett (Q uiquadrado=2.184,4; $p<0,001)$. Com a finalidade de escolher a estrutura empírica mais adequada aos dados, foram realizadas a análise do screepld, a análise dos autovalores (engenvalues) superiores a 1, a análise paralela proposta por Enzmann (1997) e a análise da consistência teórica. A análise apontou sete fatores com autovalor superior a 1, 0 screeplot e a análise paralela sugeriram a extração de cinco fatores. Portanto, a estrutura unifatorial apresentada por Caetano (1989) não se confirmou para a amostra brasileira.

Considerando os critérios de extração indicados pela análise paralela e pelo screpldt, foi testada a solução com cinco fatores. Tal solução não se mostrou consistente, em razao do agrupamento estatístico dos itens, que se concentrou em três fatores, ficando dois fatores com poucos itens, com cargas fatoriais baixas e índices de confiabilidade abaixo do limite aceitável. Além disso, a divisão em cinco fatores conduzia a um agrupamento de itens que não apresentava consistência teórica. Diante desses resultados, considerados os dados psicométricos e a consistência teórica, a configuração final foi de uma escala com 19 itens, divididos em três fatores, um deles com grau de precisão abaixo do ideal $(0,55)$. Vale destacar que, para todos eles, foram testados 0 alfa de Cronbach e 0 lambda 2 de Gutman, sem diferenças entre eles, razão pela qual somente 0 alfa de Cronbach será apresentado. Os resultados da estrutura fatorial, com os itens que compõem cada fator e suas respectivas cargas fatoriais, são apresentados na Tabela 1.

Tabela 1. Solução fatorial para a Escala de Senso de Humor na amostra brasileira

\begin{tabular}{|c|c|c|}
\hline Fatores & Itens & $\begin{array}{c}\text { Carga } \\
\text { fatorial }\end{array}$ \\
\hline \multirow{9}{*}{$\begin{array}{l}\text { Fator } 1 \text { - Humor } \\
\text { com amigos e } \\
\text { colegas }(\alpha=0,70)\end{array}$} & $\begin{array}{l}\text { 1. Se você estivesse fazendo compras numa cidade distante e, inesperadamente, } \\
\text { visse um colega de escola (ou de trabalho). }\end{array}$ & 0,65 \\
\hline & $\begin{array}{l}\text { 2. Se você fosse acordado de um sono profundo no meio da noite com o } \\
\text { telefone tocando, e se tratasse de um velho amigo que estava apenas de } \\
\text { passagem pela cidade e que decidiu telefonar para cumprimentá-lo. }\end{array}$ & 0,53 \\
\hline & $\begin{array}{l}\text { 4. D epois de ter realizado uma atividade física prolongada (ex: nadar, correr, } \\
\text { etc.), você e seus amigos estão completamente exaustos. }\end{array}$ & 0,53 \\
\hline & $\begin{array}{l}\text { 5. Se você chegasse numa festa e percebesse que outra pessoa estava usando uma } \\
\text { roupa igual a sua. }\end{array}$ & 0,37 \\
\hline & $\begin{array}{l}\text { 6. Se um amigo lhe desse um desafio (de lógica, cubo mágico, charada...) muito } \\
\text { difícil para resolver e você, para surpresa dele, fosse capaz de resolvê-lo muito } \\
\text { rapidamente. }\end{array}$ & 0,57 \\
\hline & $\begin{array}{l}\text { 7. Num dia em que você não tivesse nenhum compromisso relevante, e pudesse } \\
\text { fazer alguma coisa de que realmente gosta com alguns amigos, como você se } \\
\text { sentiria? }\end{array}$ & 0,71 \\
\hline & $\begin{array}{l}\text { 9. Se você estivesse vendo um filme ou programa de TV com alguns amigos e } \\
\text { achasse uma cena particularmente engraçada, mas ninguém mais parecesse achar } \\
\text { graça. }\end{array}$ & 0,44 \\
\hline & $\begin{array}{l}\text { 10. Se você estivesse passando uma noite romântica com alguém de quem } \\
\text { realmente gosta, por exemplo, um(a) ex-namorado(a). }\end{array}$ & 0,54 \\
\hline & $\begin{array}{l}\text { 12. Você teve a impressão de reconhecer um amigo numa sala cheia de gente. } \\
\text { Acenou para a pessoa e foi em sua direção, mas quando chegou perto dela } \\
\text { descobriu que estava enganado e que esta pessoa lhe era totalmente } \\
\text { desconhecida. }\end{array}$ & 0,50 \\
\hline Fator 2 - & 22. O seu humor é influenciado pelas pessoas com quem você está? & 0,80 \\
\hline Influências sobre 0 & 23. O seu humor é influenciado pelo lugar em que você está? & 0,83 \\
\hline humor $(\alpha=0,70)$ & 24. O seu humor é influenciado por como vocề se sente no dia? & 0,67 \\
\hline
\end{tabular}


Tabela 1. Solução fatorial para a Escala de Senso de Humor na amostra brasileira

(continuação)

\begin{tabular}{|c|c|c|}
\hline Fatores & Itens & $\begin{array}{l}\text { Carga } \\
\text { fatorial }\end{array}$ \\
\hline \multirow{7}{*}{$\begin{array}{l}\text { Fator } 3 \text { - Humor } \\
\text { em situações } \\
\text { adversas } \\
(\alpha=0,55)\end{array}$} & 3. Você acidentalmente se feriu e tem de passar alguns dias de cama. & 0,49 \\
\hline & $\begin{array}{l}\text { 8. Você estava viajando de carro num dia chuvoso, e de repente o carro derrapou } \\
\text { e ficou virado na contramão. Ninguém se feriu e o carro não sofreu dano algum. }\end{array}$ & 0,36 \\
\hline & $\begin{array}{l}\text { 11. Se você recebeu inesperadamente uma nota baixa numa prova e, mais tarde, } \\
\text { nesta noite, estivesse contando o acontecimento a um amigo. }\end{array}$ & 0,41 \\
\hline & $\begin{array}{l}\text { 13. Se você estivesse almoçando num restaurante com alguns amigos e o garçom } \\
\text { acidentalmente entornasse uma bebida sobre você. }\end{array}$ & 0,51 \\
\hline & $\begin{array}{l}\text { 14. Se você estivesse atravessando uma rua, na faixa de pedestres, e um } \\
\text { motorista impaciente, que devia ter parado, buzinasse. }\end{array}$ & 0,52 \\
\hline & $\begin{array}{l}\text { 15. Se ocorresse um problema no seu computador e você tivesse que perder toda } \\
\text { a manhã de pé, em filas, em vánias lojas de assistência técnica, tentando } \\
\text { conseguir uma solução para o problema. }\end{array}$ & 0,65 \\
\hline & $\begin{array}{l}\text { 17. Se o seu(sua) namorado(a) tivesse decidido romper com você por ter } \\
\text { encontrado outra pessoa e, alguns dias depois, você contasse a um grande amigo } \\
\text { o que lhe aconteceu. }\end{array}$ & 0,64 \\
\hline
\end{tabular}

Tabela 2. Descrição dos fatores da Escala de Senso de Humor no Brasil

\begin{tabular}{llcrc}
\hline Fatores & Descrição & Mín - Máx. & Méáa & DP \\
\hline $\begin{array}{l}\text { Fator 1 - Humor com } \\
\text { amigos e colegas }\end{array}$ & $\begin{array}{l}\text { Senso de humor demonstrado em situações que } \\
\text { envolvem amigos e colegas. }\end{array}$ & $1,1-4,8$ & 2,96 & 0,59 \\
\hline $\begin{array}{l}\text { Fator 2 - Influências } \\
\text { sobre o humor }\end{array}$ & $\begin{array}{l}\text { Grau em que o humor é afetado considerando-se as } \\
\text { mudanças situacionais relativas a localização, } \\
\text { companhia e sentimentos momentâneos. }\end{array}$ & $1,0-5,0$ & 3,27 & 0,97 \\
\hline $\begin{array}{l}\text { Fator 3 - Humor em } \\
\text { situações adversas }\end{array}$ & $\begin{array}{l}\text { Senso de humor demonstrado em situações adversas } \\
\text { que não envolvem pessoas conhecidas. }\end{array}$ & $1,0-3,6$ & 1,31 & 0,39 \\
\hline
\end{tabular}

Os valores médios obtidos em cada um desses fatores sinalizam para um maior senso de humor dos brasileiros com situações que envolvam amigos e colegas do que com pessoas desconhecidas e para uma média relativamente alta para possíveis variações de humor de acordo com o local, as pessoas presentes ou a forma como a pessoa se sente naquele dia. A Tabela 2 mostra resultados de estatística descritiva para os fatores, além de uma descrição para cada um deles.

No que se refere à correlação entre os três fatores da escala, houve correlação significativa $(p \leq 0,05)$ entre os fatores "humor com amigos e colegas" e "humor em situações adversas" ( $r=0,30)$ e o fator "influências sobre o humor" não teve correlação com nenhum dos outros fatores, o que sugeriu a utilização da técnica de rotação oblíqua. A respeito desses resultados, vale registrar que a correlação obtida entre "humor com amigos e colegas" e "humor em situações adversas" foi uma correlação que pode ser considerada de fraca a moderada na classificação de Miles e Shevlin (2001). Tal resultado poderia ser esperado, uma vez que a escala mede a expressão do humor em diferentes situações e, de fato, situações vivenciadas com amigos e colegas costumam ser situações descontraídas e de prazer, enquanto o outro fator refere-se a situações adversas, nas quais o humor se manifesta, realmente, de forma diferenciada.

Outro aspecto que merece discussão diz respeito ao fator "influências sobre 0 humor". Esse fator mensura o grau em que o humor é afetado, considerando-se as mudanças situacionais relativas a localização, companhia e sentimentos momentâneos. Nesse sentido, talvez fosse mais adequado considerar esses itens como compondo uma escala separada, que aponta o grau de variabilidade do humor e não a quantidade em que ele ocorre nas diferentes situações. A diferença do enfoque do que está sendo medido é a explicação mais provável para a ausência de correlação entre o fator "influências sobre o humor" e os outros dois fatores.

O bservou-se que, para a formação da versão final dessa escala, o processo empírico eliminou cinco itens complexos, que tinham cargas significativas em dois fatores ou que não apresentavam cargas acima de 0,32 em nenhum fator. A variância total explicada dos três fatores foi de 31,3\%, sendo, respectivamente, as variâncias explicadas por cada um dos fatores equivalentes a: 15,0; 8,7 e 7,7\%. Esperava-se uma variância total explicada maior, uma vez que 0 instrumento continha em sua versão original um 
número maior de fatores. Assim, novas testagens dessa escala, com a inclusão de novos itens específicos de humor situacional, considerando as especificidades da cultura brasileira podem ser úteis para possibilitar a ampliação da variância total explicada pelo instrumento.

Para checagem da estrutura final da escala, foram calculados os índices de precisão da escala (alfa de Cronbach e lambda 2 de Gutman) se deletado cada um dos itens dos seis fatores extraídos. 0 resultado apontou que, qualquer que fosse 0 item retirado, haveria uma diminuição do grau de precisão, confirmando a pertinência de manutenção dos 19 itens da versão final da escala. A única exceção refere-se ao item "o seu humor é influenciado por como você se sente no dia", cuja exclusão resultaria em um aumento de duas casas decimais no valor dos indicadores de precisão, porém, nesse caso, o fator ficaria com apenas dois itens, o que não seria uma solução recomendável.

Em relação à diferença de média de humor encontrada entre homens e mulheres na pesquisa de Caetano (1989), em Portugal, e não encontrada na validação original da escala feita por Martin e Lefcourt (1984), observou-se um resultado no qual os fatores "humor com amigos e colegas" e "influências sobre 0 humor" não apresentaram médias signficativamente diferentes por gênero. Já o fator "humor em situações adversas" apresentou diferença significativa (t[617]= $3,52 ; \mathrm{p}<0,001)$ entre homens e mulheres na amostra brasileira, estas apresentando uma média de humor ligeiramente mais baixa $(1,26)$ do que aqueles $(1,39)$.

Um dado interessante refere-se ao item 20 do instrumento, 0 qual media a autoclassificação quantitativa de humor. Em estudos anteriores (Caetano, 1989; Martin \& Lefcourt, 1984), com amostras canadenses e portuguesas, as médias foram muito mais elevadas do que as obtidas no Brasil. Enquanto no Canadá e em Portugal obtiveram-se $94 \%$ e $91,2 \%$, respectivamente, de respostas nas quais a pessoa se via com um senso de humor na média ou acima da média, no Brasil esse percentual é muito inferior (54,8\%). Tal resultado pode indicar uma diferença cultural, quer seja no grau de senso de humor, quer seja na concepção do que seja a "média" do humor nos grupos em que as pessoas convivem, ou ainda, uma maior ou menor influência do efeito de desejabilidade social. Seria interessante que outras pesquisas fossem realizadas para aprofundar esta discussão.

O processo de adaptação transcultural resultou na versão em português do instrumento denominado Escala de Senso de Humor Situacional (ESHS). O instrumento traduzido inclui instruções para sua utilização e mantém a apresentação do instrumento original. Esse instrumento validado permite 0 desenvolvimento de novas pesquisas sobre senso de humor, incluindo a possível definição de pontos de corte do grau de humor. Estudos dessa natureza podem ser bastante úteis para diversas áreas da psicologia.

\section{Conclusões}

Há uma sensível carência de instrumentos que possam mensurar emoções de um modo geral, sendo raros os estudos sobre o humor situacional. Nesse sentido, a presente pesquisa dá uma contribuição ao apresentar uma forma de mensuração de uma variável pouco pesquisada, mas bastante presente e possivelmente influente no dia a dia das pessoas.

A versão traduzida para o português do Brasil do Situational Humr Response Questionaire (SHRQ), intitulada Escala de Senso de Humor Situacional (ESHS), demonstrou possuir evidências de validade psicométrica, uma vez que as cargas fatoriais dos itens ficaram acima de 0,35 (nos três fatores) e que o grau de precisão (alfa de Cronbach) indicou valores adequados para dois dos fatores $(0,70)$, sendo indicado ampliar 0 número de itens do terceiro fator ("humor em situações adversas" ) para melhoria do grau de precisão obtido $(0,55)$.

Esses resultados indicam que a escala pode ser utilizada para comparar grupos ou para análises individuais, com grande gama de aplicação nas diversas áreas de atuação da psicologia. Pesquisas futuras podem focar tanto na análise dos determinantes do humor situacional como dos efeitos que essa característica pode ter sobre aspectos diversos da vida das pessoas. Como as respostas ao instrumento em Portugal e no Brasil mostram que o humor é uma característica individual positivamente valorada pela maioria das pessoas, recomenda-se que o uso da escala seja feito com delineamentos experimentais que permitam o controle de um possível efeito de desejabilidade social.

Como limitações do estudo é preciso registrar 0 fato de não ter sido realizado teste e reteste do instrumento. Outra limitação que pode ser mencionada refere-se ao fato de a metade da amostra ser de universitários, indicando-se a aplicação do instrumento em contextos diversificados. Seria também aconselhável a aplicação desta escala de humor em conjunto com outras escalas com 0 objetivo de investigar a validade de construto. 


\section{Referências}

Brief, A. P., \& Weiss, H. M. (2002). Organizational behavior: affect in the workplace. Annul Review Psychdogy, 53, 279-307.

Caetano, A. (1989). Medida quantitativa do humor: validação de um questionário. Psicdoga, VII(3), 285-290.

Crocker, L., \& Algina, J. (1986). Introdurtion to dassical and modem teet theery. O rlando: Harcourt Brace Jovanovich College Publishers.

Cunha, J. A. (2001). Manual da vesão em patughếs das Escalas Bekk. São Paulo: Casa do Psicólogo.

Del Nero, H. S. (1997). O śtio da mente pensamento, emoção e vontade no cédbro humano São Paulo: Collegium Cognitio.

Dixon, N. R. (1980). Humor: a cognitive altemative to stress. Stress andAnxidy, 7, 281-289.

Duncan, W. J. (1984). Perceived humor and social network patterns in a sample of task-oriented groups: a reexamination of prior research. Human Rdations, 37(11), 895-907.

Enzmann, D. (1997). RanEigen: a program to determine the parallel analysis criterion for the number of principal components. Applied Psychdogical Measurement, 21, 232.

Estrada, C. A., Isen, A. M., \& Young, M. J. (1997). Positive affect facilitates integration of information and decreases anchoring in reasoning among physicians. Organizational Benavior and Human Deision Proseses 72, 117-135.

George, J. M. (1989). Mood and absence. Jaumal of ApplielPsychdogy, 74(2), 317-324.

Gondim, S. M. G., \& Siqueira, M. M. M. (2004). Emoções e afetos no trabalho. Em J. C. Zanelli, J. E. Borges-Andrade \& A. V. B. Bastos (Eds.), Psicdoga, Organizaçoes eTrabalho no Brasil (pp. 207236). Porto Alegre: Artmed.

Gray, E., \& Watson, D. (2001). Emotion, mood and temperament: similarities, differences and a synthesis. Em R. L. Payne \& C. L. Cooper (Eds.), Emations at work: theory, rearch and applications for managment (pp. 21-44). Londres: John Wiley \& Sons.

Guillemin, F. (1995). Cross-cultural adaptation and validation of health status measures. Scandinavian Jamal of Rheumatdogy, 24(2), 61-63.
Houzel, H. S. (2002). O ć́dbronossodecada dia (4a․ ed.). Rio de Janeiro: Viera \& Lent.

Isen, A. M. (1999). Positive affect, cognitive-processes, and social-behavior. Advanres in Experimental Social Psydhlogy, 20, 203-253.

Madjar, N., Oldham, G. R., \& Pratt, M. G. 2002. There's no place like home? The contributions of work and non-work creativity support to employee's creative performance. Academy of ManagementJaumal, 45, 757-767.

Martin, R. A., \& Lefcourt, H. M. (1984). Situacional humor questionnaire: quantitative measure of sense of humor. Jaumal of Pesonality and Social Psychdogy, 47(1), 145-155.

Miles, J., \& Shevlin, M. (2001). Applying Regession and Comdation: a guide for students Londres: Sage Publications.

Morrell, J. (1997). Humr warks Amherst, Massachusetts: HRD.

Pasquali, L. (1999). Instrumentos psicdógicos manual prático dedaborasãa Brasília: LabPAM/ UnB.

Pasquali, L. (2003). Psicomeria: teria dostestes na psicdoga ena educacãa Petrópolis: Vozes.

Pelled, L, \& Xin, K. (1999). Down and out: an investigation of the relationship between mood and employee withdrawal behavior. Jaumal of Managemet, 25(6), 875-895.

Pillutla, M. M., \& Murnighan, J. K. (1996). Unfairness, anger, and spite: emotional rejections of ultimatum offers. Organizational Behavior Human Deision Process, 68, 208-224.

Sperber, A. D. (2004). Translation and validation of study instruments for 15. cross-cultural research. Gastroentedogy, 126(1 Suppl 1), S124-128.

Silva-Filho, A. C. P. (2003). Psicanálise e neurociências. Reista dePsiquiatria Clínica, 30(3), 104-107.

Tabachnick, B. G., \& Fidell, L. S. (2007). Using miltivariate statistics (5 $5^{\underline{a}}$ ed.). Boston: Pearson Education. 
Sobre as autoras:

Adriana Benevides Soares é psicóloga, doutora em Psicologia Cognitiva pela Universidade de Paris XI, docente da graduação e da pós-graduação da Universidade Salgado de Oliveira e D ocente da graduação da Universidade do Estado do Rio de Janeiro.

Luciana Mourão é comunicadora social e administradora, docente da graduação e da pós-graduação da Universidade Salgado de Oliveira e docente de graduação da Associação Internacional de Educação Continuada AIEC.

Contato com as autoras:

Universidade Salgado de Oliveira. Rua Marechal Deodoro, 263 - Centro - CEP 24030-060. Rio de Janeiro-RJ.

E-mail: adribenevides@gmail.com 